revista ANTHROPOLÓGICAS

Ano 24, 31(2): 28-60, 2020

\title{
Políticas e Poéticas da Infraestrutura
}

Brian Larkin ${ }^{a}$

Infraestruturas são formas materiais que permitem trocas por meio do espaço. Elas são redes físicas através das quais mercadorias, ideias, lixo, energia, pessoas e capitais financeiros circulam. Neste artigo, mapeio a literatura antropológica que busca teorizar a infraestrutura a partir da biopolítica, dos estudos de ciência e tecnologia e das teorias de tecnopolítica. Também examino outros aspectos das infraestruturas que proporcionam diferentes significados e estruturas políticas por meio das dimensões estética, sensorial, do desejo e da promessa.

Infraestrutura, Tecnopolítica, Biopolítica, Estética e Tecnologia.

Infraestruturas ${ }^{1}$ são redes construídas para facilitar o fluxo de bens, pessoas e ideias, permitindo o intercâmbio dos mesmos no espaço. Enquanto formas físicas, moldam a natureza de uma rede, a velocidade e a direção de seus movimentos, assim como sua temporalidade e sua vulnerabilidade às falhas ${ }^{2}$. Elas constituem a arquitetura de circulação que, literalmente, sustenta as sociedades modernas e, consequentemente, cria o ambiente das interações da vida cotidiana. A antropologia, até recentemente, teve pouco a dizer sobre as infraestruturas, porém, na última década, novas direções intelectuais na disciplina começaram a dar centralidade à questão das infraestruturas.

$\overline{\text { a Departamento }}$ de Antropologia, Columbia University. Email: bl190@columbia.edu. 
Neste artigo, avalio o que uma análise das infraestruturas oferece à análise antropológica e o que a antropologia acrescenta no estudo das infraestruturas.

Ainda que a antropologia tenha lidado por anos com a metáfora da infraestrutura para se referir a qualquer sistema que pareça sustentar ou incitar o mundo dos fenômenos (cultura, episteme, estrutura social), desde uma análise marxista das relações entre a base e a superestrutura até a distinção saussuriana entre langue e parole, pode-se argumentar que a antropologia encontra dificuldades - etnograficamente - para analisar sistemas tecnológicos em si. A inclinação da nossa disciplina é analisar a influência de uma estrada em uma região do Peru (Harvey 2012) ou da Nigéria (Masquelier 2002) em vez de analisar a construção de estradas enquanto uma rede ${ }^{3}$. Nesse sentido, uma análise desses sistemas requer uma reorganização metodológica na qual a etnografia pode ser conduzida em centros governamentais normalmente distantes de onde a estrada está sendo construída e, portanto, podendo levar em conta políticos, tecnocratas, economistas, engenheiros e construtores de estradas, assim como os próprios usuários das estradas. Porém, como notou Latour (1993) já há algum tempo, essa abordagem é tanto uma força quanto uma fraqueza para a disciplina, por colocar em destaque outras áreas nas quais as infraestruturas se espalham. Isso possibilita compreender o papel dos colapsos e das formas de vida que daí se originam (Kockelman 2010; Larkin 2008). A antropologia certamente precisa compreender a racionalidade para propor uma etnografia de infraestruturas, e aqui me refiro a alguns dos melhores trabalhos que seguem nessa direção (Appel 2012b; Anand 2011; Bear 2007; Collier 2011; Von Schnitzler 2008). Contudo, é necessário que a antropologia mantenha o foco nas suas possibilidades, ou seja, nas maneiras em que formas de infraestruturas podem oferecer intuições sobre outros domínios, tais como práticas de governo, de religião ou de socialidade.

Talvez a abordagem mais dinâmica na antropologia contemporânea para estudar infraestruturas tenha vindo com o conceito de tecno- 
política. Como muitos estudiosos têm apontado, o liberalismo é uma forma de governo que renega a si mesma a possibilidade de adotar lógicas para pensar populações e territórios, embasadas em domínios tecnológicos que geralmente não parecem se relacionar às instituições políticas formais (Barry 2001; Joyce 2003; Mitchell 2002, 2011). Mesmo o livre fluxo de mercadorias que constitui a economia liberal apoia-se em uma base infraestrutural que organiza mercado e sociedade. Vários pesquisadores têm procurado recorrer a estudos científicos e tecnológicos para rastrear as operações materiais dessas tecnologias e para entender as suas consequências nos processos políticos (Bennet 2010; Callon 1998; Latour 2007; Mitchell, 2011). Nesses trabalhos, as infraestruturas são relevantes por revelarem as formas de racionalidade política que fundamentam os projetos tecnológicos e que dão origem a um "aparato de governamentalidade" (Foucault 2010:70).

Contudo, as infraestruturas existem para além do funcionamento puramente técnico, elas precisam ser analisadas enquanto veículos semióticos e estéticos orientados para os seus destinatários. Elas fazem emergir e simultaneamente armazenam em si formas de desejos e fantasias, podendo então assumir aspectos fetichistas que, ocasionalmente, podem ser completamente independentes das suas funções técnicas. Focar na questão da forma ou da poética das infraestruturas permite-nos compreender como o político pode ser constituído por meio de diferentes significados. Isso aponta para sentidos de desejo e de possibilidade, o que Benjamin (1999) chamaria de fantasia coletiva da sociedade (De Boeck 2011; Humphery 2005; Khan 2006; Larkin 2008; Mzárek 2002; Sneath et al 2009). Significa também estar consciente das dimensões formais das infraestruturas, compreendendo qual tipo de objetos semióticos elas são, as suas operações técnicas, e determinando como elas endereçam e constituem sujeitos.

\section{A ontologia da infraestrutura}

Infraestruturas são matérias que possibilitam o movimento de outras matérias. Sua peculiar ontologia situa-se no fato de que elas são 
coisas e, ao mesmo tempo, são relações entre coisas. Enquanto coisas, oferecem-se aos sentidos, porém, ainda assim, deslocam o foco para os assuntos que movimentam. Nós geralmente vemos computadores, não cabos; vemos luz, não eletricidade; vemos torneiras e água, mas não canos e esgotos. Enquanto objetos técnicos, eles precisam ser examinados por meio da extensa tradição teórica sobre tecnologia (Giedion 1969; Heidgger 1977; Marx 1990; Mumford 2010; Simondon 1980 [1958]; Stiegler 1998). Contudo, a dualidade das infraestruturas indica que, na medida em que esses objetos técnicos operam sistematicamente, elas não podem ser teorizadas como um objeto isolado. $\mathrm{O}$ que distingue as infraestruturas das tecnologias é o fato de serem constituídas por objetos que suportam a operatividade de outros objetos e, ao fazê-lo, operam como sistemas ${ }^{4}$.

Talvez, devido a essa dualidade, as infraestruturas dificilmente são governáveis por conceitos. Elas são pensadas normalmente como "sistemas de substratos" (Star 1990:380) que subjazem um mundo fenomenológico construído com canos, cabos esgotos e fios. Essa visão presume uma relação clara e linear entre um sistema de substratos e um mundo fenomenológico ao qual dá origem, enquanto a definição da relação entre os dois torna-se mais e mais difícil. Tomemos como exemplo o computador que usei para escrever este artigo. Qual a sua infraestrutura? Eletricidade é provavelmente o mais óbvio substrato que permite o funcionamento do computador. Porém, como notou Edwards (1998), mesmo que a eletricidade seja a infraestrutura do computador, o computador é a infraestrutura para o fornecimento de energia, já que toda transmissão é regulada por computadores. A eletricidade, por sua vez, está associada a outras infraestruturas, entre as quais podemos incluir a imprescindível produção de petróleo (Appel 2012a e 2012b; Gelber 2015) para o funcionamento das usinas elétricas; os mecanismos financeiros conseguintes à descentralização que permitem a venda de eletricidade no livre mercado; ou ainda as redes de trabalho que permitem a sua produção e distribuição. 
Nosso estudo de infraestrutura poderia então focar nas coisas construídas e nas coisas relacionadas ao conhecimento e às pessoas. A partir da teoria ator-rede, é possível analisar todos esses elementos distintos como um só sistema, porém a questão de quais elementos estão inclusos e quais estão excluídos do sistema é mantida. Afinal, a eletricidade é somente uma das várias infraestruturas que dão suporte ao funcionamento dos computadores: há ainda o sistema telemático que permite transmitir e receber informação (Graham \& Marvin 1996), os protocolos dos softwares que delimitam as possibilidades de uso das máquinas (Chun 2008; Galloway 2006), e as competências educacionais e culturais necessárias ao entendimento das funcionalidades do sistema e de como operá-lo. Todos esses substratos são indispensáveis para o computador funcionar. A relação linear e simples, fundamentada nos objetos visíveis, acaba sendo recursiva e dispersa. Dada a constante proliferação das redes que podem ser mobilizadas para a compreensão das infraestruturas, somos lembrados que debater sobre uma infraestrutura é um ato categórico. É um momento de expor essas redes heterogêneas para definir quais elementos delas devem ser discutidos e quais devem ser ignorados. Reconhece-se que as infraestruturas operam em diferentes níveis simultaneamente, produzindo múltiplos direcionamentos e levando a pensar que qualquer arranjo específico de questões intelectuais irá determinar quais desses níveis serão examinados. As infraestruturas não são algo que, em um sentido positivista, simplesmente estão 'lá'. $O$ ato de definir uma infraestrutura é um momento categórico. Tomada de modo refletido, elas comportam uma analítica cultural que enfatiza os comprometimentos epistemológicos e políticos envolvidos na seleção do que é visto como infraestrutural (e, portanto, causal) e daquilo que é deixado de fora.

\section{Pensamentos sistemáticos e tecnopolítica}

Já faz algum tempo que intelectuais ligados aos estudos de ciência e tecnologia e à geografia têm analisado como as infraestruturas mediam trocas à distância, colocando diferentes pessoas, objetos e espa- 
ços em interação e conformando, assim, a base de funcionamento dos sistemas econômicos e sociais modernos (Graham \& Marvin 1996; 2001; Lefebvre 1991). Graham \& Marvin (1996) têm escrito uma série de influentes trabalhos examinando como os novos sistemas de telecomunicações estão reconfigurando os espaços urbanos e como as infraestruturas agregam água, energia, pessoas e ruas em um conjunto de infraestruturas interconectadas que definem a vida moderna (2001). A ênfase aqui recai sobre a formação daquilo que Hughes (1987; 1993) tem chamado de sistemas técnicos de larga escala, as gigantescas redes de infraestruturas que organizam a vida cotidiana. Hughes $(1987$; 1993) e outros autores seguindo sua direção (Bijker 1997; Bijker et al 1987; Bowker \& Star 2000; Edwards 1997, 2003; Edwards et al 2009; Hecht 2011; Jackson et al 2007; Star \& Ruhleder 1996; Yates 1993) argumentam que as infraestruturas tipicamente começam com uma série de tecnologias pequenas e independentes com uma ampla variedade de padrões técnicos. Elas tornam-se infraestruturas seja na medida em que um sistema tecnológico se torna dominante sobre os outros, seja quando os sistemas independentes convergem em uma rede. Enquanto um teórico dos sistemas, Hughes considera que a ontologia da tecnologia é composta de elementos internos aos próprios objetos, mas que, ao mesmo tempo, são partes de um único sistema. Desse modo, a invenção de Edison da lâmpada envolve simultaneamente outras de suas invenções, como o gerador e o alimentador $^{5}$. Tais invenções permitiram uma inovação nos instrumentos financeiros e nas estruturas administrativas necessárias para acomodar o rápido crescimento corporativo. Nessa concepção, a lâmpada não se desenvolve a partir dos elementos internos à própria tecnologia (uma associação de vidro, filamentos e vácuo), como algumas teorias da técnica apontariam. Em vez disso, enquanto infraestrutura, ela é um amálgama de expertises técnicos, administrativos e financeiros.

Duas grandes diretrizes de fundamental importância para a antropologia emergem desses trabalhos. Colocar o sistema no centro da análise descentra o foco da tecnologia e oferece uma perspectiva mais 
sintética, que inclui na nossa concepção de máquinas todo tipo de elemento não tecnológico. Para Hughes, uma empresa ou uma prática de contabilidade são invenções técnicas tanto quanto o são um dínamo ou um telefone, e todos esses elementos são essenciais para produzir um sistema infraestrutural. Em segundo lugar, podemos considerar o foco sobre a construção dos sistemas. Um sistema técnico origina-se em um lugar e cresce em resposta a técnicas específicas comuns na área em que o sistema é desenvolvido, que podem ser ecológicas, legais, políticas e industriais. Porém, na medida em que cresce dentro de uma infraestrutura em rede, o sistema deve se mover para outros lugares com diferentes condições, padrões tecnológicos e regulamentações legais, adotando então técnicas de adaptação e tradução. Colocando o foco sobre as práticas de rotinização e expansão, torna-se nítida a exigência da tradução (podendo ser técnica, mas também administrativa e financeira) enquanto um processo inerente à construção do sistema. Não surpreende que os intelectuais que vieram depois de Hughes (Bowker \& Star 2000; Edwards et al 2009) valeram-se fortemente da teoria ator-rede e de suas ênfases na descrição das associações entre redes heterogêneas (Latour, 1996; 2007) e na necessidade de levar em conta como a tradução ocorre (Callon 1986; 1998; Latour 1993). Esse esforço tem sido extremamente produtivo para a pesquisa antropológica recente (Appel 2012a e 2012b; Anand 2011, 2012; Carse 2012; Collier 2011; Collier \& Lakoff 2008; Von Schnitzler 2008), como se pode notar em dois excelentes trabalhos sobre abastecimento de água em Mumbai (Anand 2011, 2012) e em Soweto (Von Schnitzler 2008).

A etnografia de Anand reúne engenheiros, articuladores políticos, moradores de comunidades pobres, políticos, ativistas e burocratas dentro de um único sistema de operações técnicas que permitem o abastecimento de água. (ver também Gandy 2008; Graham et al 2013). Em Mumbai, a densidade populacional faz com que o abastecimento de água seja escasso e intermitente, e os engenheiros do município tratam essa limitação em termos técnicos (falam da localização das comunidades pobres no topo dos morros e da pressão necessária 
para movimentar a água por grandes distâncias). Porém, para os residentes desses lugares, essa é uma questão de mobilização política. As comunidades sem água envolvem-se com os dadas, poderosos patronos que usam suas conexões para pressionar políticos eleitos a fornecer conexão infraestrutural. Em troca, o dada recompensa-os com suporte eleitoral.

$\mathrm{Na}$ análise de Anand, interagem basicamente dois sistemas infraestruturais: a distribuição de água com seus canos, os engenheiros e a burocracia constituem o aspecto técnico do fornecimento (o abastecimento infraestrutural o qual constitui todo o sistema de larga escala para Hughes e outros); e, do outro lado, as redes sociais, as formas de clientelismo e o 'trabalho fático' (Elyachar 2010), tão importantes para a distribuição de água quanto as bombas e os canos. Nesse sentido, infraestrutura é um tipo de mentalidade e uma forma de viver no mundo (Hansen \& Verkaaik 2009; Simmel 1972; Simone 2001), e Anand (2011) faz convergir essas duas concepções diferentes de infraestrutura, não para analisar o abastecimento de água, mas para revelar a produção do que o autor considera uma "cidadania hidráulica, uma forma de pertencimento à cidade que é possibilitada por reivindicações sociais e materiais voltadas à infraestrutura de água” (Anand 2011:545).

Von Schnitzler (2008), assim como Anand, vê o abastecimento de água enquanto algo revelador de uma lógica maior de cidadania na África do Sul pós-apartheid. Porém, a abordagem dela difere da análise anterior por focar em uma tecnologia específica, o hidrômetro, introduzido como um dispositivo técnico para monitorar o uso de água e acabar com o desperdício nas townships ${ }^{6}$. Von Schnitzler descreve a história técnica do hidrômetro, desde sua invenção na Grã-Bretanha até sua introdução na África do Sul, para então pensar como não se tratava somente de regular o uso da água nesse contexto, mas de produzir um novo tipo de cidadania. O cidadão era o responsável, monitorando por si suas ações e engajando-se em práticas de contabilização. Voltando-se mais para a biopolítica, Von Schnitzler argumenta 
que os hidrômetros não regulam somente a água, eles revelam uma estratégia de governo e, em última instância, produzem uma ética. "[E] scondendo-se atrás de uma linguagem pedagógico-moral" (Von Schnitzler 2008:906), os funcionários públicos da cidade tinham como objetivo reformar a cultura dos residentes dos subúrbios (que desperdiçavam água) produzindo assim um 'sujeito calculista' que fecha a torneira enquanto escova os dentes ou reutiliza a água do banho para dar a descarga no banheiro (ver também Fennell 2011). O hidrômetro, argumenta Von Schnitzler, distribuía tanto um comportamento moral quanto a água. Ambos, Von Schnitzler e Anand, demonstram como o funcionamento das tecnologias, apesar da aparente neutralidade prática, torna-se a base ao redor da qual formas de cidadania são disputadas (Barry 2001; Joyce 2003; Mitchell 2002, 2011; Otter 2008) e, além disso, é o ponto em que os sistemas tecnológicos entrelaçam-se com outros domínios, tais como religiosos e políticos.

Collier acrescenta uma torção nessa literatura ao organizar sua análise das infraestruturas inteiramente em torno da questão da biopolítica (Foucault 2010, 2011). Para Collier, as infraestruturas são uma mistura de racionalidade política, técnicas administrativas e sistemas materiais, e o seu interesse não é a infraestrutura em si, mas o que ela pode dizer sobre práticas de governo. Através dessas lentes, a análise do fornecimento de eletricidade na União Soviética revela um sistema totalizante em uma economia planificada pelo governo, em vez de exibir os efeitos da eletricidade para os usuários. Diferente do Oeste, na União Soviética, o fornecimento de eletricidade não era regulado pela demanda do usuário. Sendo parte de um sistema totalizante de planejamento da eletricidade, esta era previamente alocada para determinadas regiões por decisão dos tecnocratas. $\bigcirc$ processo de transição para a Rússia pós-soviética exigiu um desmantelamento dessa ideia de governo por um conceito de sociedade organizada em torno do consumidor individual e de suas próprias demandas, em vez de coletivos, aos quais são designados quantidades fixas de eletricidade. 
Collier (2011) redireciona a análise em uma contracorrente. Distanciando-se dos efeitos sociais da infraestrutura, o autor vai na direção das práticas conceptualizadas que surgem antes da construção dos próprios sistemas e que são projetadas neles. No fim, seu interesse na infraestrutura é pelo fato de ela permitir o rastreio da transposição e a operacionalização de teorias econômicas surgidas na América neoliberal e importadas para a Rússia no começo da transição pós-soviética. A infraestrutura torna-se a evidência material dessa transposição. Estudar infraestruturas etnograficamente, para Collier, é fazer da teoria econômica um objeto etnográfico tão material quanto os canos ou as relações sociais às quais dão origem. É uma etnografia que produz diferentes objetos infraestruturais, em que o orçamento como locus de racionalização formal é tão importante quanto o material bruto dos sistemas tecnológicos. Para Collier, ambos lançam luz sobre os estilos de pensamento e sobre as mudanças de racionalidade no governo biopolítico.

\section{A intolerável modernidade da infraestrutura}

A infraestrutura tem seus fundamentos conceituais na ideia iluminista de um mundo em movimento e aberto a mudanças, onde a livre circulação de bens, ideias e pessoas cria a possibilidade de progresso (Mattelart 1996, 2000). Esse pensamento é a razão pela qual o fornecimento de infraestruturas é tão intimamente conectado com o sentido da modelagem da sociedade e da realização do futuro. Eles são "mecanismos para controlar o tempo", escrevem Graham \& Marvin (1996:42; ver também Edwards 2003), de modo que a posse de eletricidade, as ferrovias e a água corrente passaram a definir o que seria a civilização. Nesse sentido, é muito difícil separar as infraestruturas dos modelos evolucionários de pensamento, principalmente por isso ser uma parte tão íntima de seu atrativo. Para Marx (1990), as tecnologias infraestruturais não eram simplesmente coisas materiais, mas decretavam o próprio curso da história, já que a indústria era um sistema de máquinas que deixava de lado os "males herdados" 
e as "sobrevivências" (Marx 1990:91), na medida em que o curso da história se desdobrava. As infraestruturas eram partes vitais para a organização de uma economia de mercado e para o conceito de progresso, central para o liberalismo (Foucault 2010). Aproveitar essa força da história é um poder tão belo e tão convincente que, como Freud (1989) reconheceu, atende a um desejo básico de domínio humano.

“Com todos os seus instrumentos ele aperfeiçoa os seus órgãos tanto motores como sensoriais - ou elimina os obstáculos para o desempenho deles. Os motores lhe colocam à disposição imensas energias, que, tal como seus músculos, ele pode empregar em qualquer direção; os navios e os aviões não deixam que a água e o ar lhe impeçam a movimentação. Com os óculos ele corrige as falhas da lente de seu olho, com o telescópio enxerga a enormes distâncias... Com o auxílio do telefone, ele ouve bem longe, de distâncias que seriam tidas por inalcançáveis até mesmo em contos de fadas ... O ser humano tornou-se, por assim dizer, uma espécie de deus protético, realmente admirável quando coloca todos os seus órgãos auxiliares" (Freud 2010 [1930]:33-34). ${ }^{7}$

Um resultado dessa inebriante linhagem é a dificuldade de separar as infraestruturas de sua estratificação histórica e de nossa crença nela; ao promover a circulação, as infraestruturas trazem mudanças e, por meio das mudanças, elas representam progresso, e por meio do progresso, conquistamos liberdade. Talvez esse processo explique por que, enquanto objetos, elas provoquem comprometimentos afetivos tão profundos, particularmente - mas não exclusivamente - nas sociedades em desenvolvimento. O historiador tcheco Mrázek (2002) descreve a experiência da infraestrutura como um "entusiasmo da imaginação" (Mrázek 2002:166), referindo-se aos sentimentos de promessa que as tecnologias, tal como as infraestruturas, podem estimular ${ }^{8}$. A função técnica de uma estrada é transportar veículos de um lugar ao outro, promovendo movimento e concretizando o objetivo iluminista para a sociedade e para a economia enquanto um espaço de circulação irrestrita. Mas também pode ser um objeto excessivamente fantástico, que gera desejo e pavor, independentemente da sua funcionalidade 
técnica. Muitos projetos infraestruturais são cópias, construídas e financiadas para que as cidades ou as nações, ao repetirem os projetos infraestruturais de outros lugares, possam fazer parte da modernidade contemporânea e, assim, compartilharem um paradigma visual e conceitual ligado ao significado de ser moderno. Dalakoglou (2010) refere-se a esse processo como fetichismo infraestrutural, ao escrever sobre a Albania, onde milhas de estradas vazias foram construídas mesmo que o estado comunista tenha, em grande medida, impedido a propriedade privada de carros. Pedersen (2011) faz um argumento similar sobre os investimentos russos em infrastruktura serem um pré-requisito à modernidade socialista, visando ao progresso material e, portanto, ideológico. "[I]nvestimentos em infraestrutura não era [...] racional em nenhum sentido econômico estreito; ao contrário, construir 'metrópoles em miniatura' era compreendido como investimentos num novo ser, numa nova humanidade, num novo cosmos" (Pedersen 2011:45). As ruínas de projetos de infraestrutura fracassados dão testemunho de uma certa estrutura de sentimento que é constitutiva do investimento imaginativo do estado pós-colonial em tecnologia.

Desse modo, estradas e ferroviais não são somente objetos técnicos, mas também operam em um nível de fantasia e desejo. Eles codificam sonhos de indivíduos e sociedades, sendo então os veículos por meio dos quais essas fantasias são transmitidas e tornadas reais emocionalmente. De forma similar, Benjamin, ao tratar daqueles que cresceram próximos a ferrovias, argumentou que nunca se pode analisar a coisa em si, mas, em vez disso, deve-se confrontar o passado dessas pessoas, seus próprios desejos e fantasias, que funcionam como filtros pelos quais o objeto é visto. De fato, para Benjamin, mercadorias, edifícios e ruas continham em si o movimento da história: eles seriam a materialização ${ }^{9}$ de forças históricas objetivas, mas, ao mesmo tempo, entram no nosso inconsciente e dominam a imaginação. Eles nos conformam como sujeitos não somente em um nível tecnopolítico, mas também por meio dessa mobilização de afeto e dos sentidos 
de desejo, orgulho e frustração, sentimentos que podem ser profundamente políticos.

Esse modo de operar engloba uma importante parte do direcionamento político da infraestrutura - a maneira na qual as tecnologias passam a representar a possibilidade de ser moderno e de ter um futuro ou, por outro lado, a exclusão dessa possibilidade, resultando em uma experiência de abjeção (Archambault 2012; Ferguson 1999). E isso ocorre tanto em um nível individual quanto social. Barker (2005) poderosamente transmite esse sentido em sua análise dos engenheiros indonésios envolvidos na construção do Palapa, um dos primeiros satélites lançados por uma nação em desenvolvimento. Baker escreve sobre uma 'solidariedade intensa' entre engenheiros em formação no Instituto de Tecnologia de Bandung, unidos por uma única causa. "Não havia competição entre os estudantes, pois, naquele momento, queríamos desenvolver [o membangup]" (Barker 2005:712). Sua descrição assemelha-se às entrevistas de Lombardi (1999) com os primeiros engenheiros de telecomunicações no Brasil, já que eles "afirmavam, num tom solene, a dedicação altruístas dos [...] colegas trabalhadores enquanto lutavam ... para manter o Brasil na vanguarda do progresso da telefonia" (Lombardi 1999:21). Minha própria pesquisa sobre a história dos sistemas mídia na Nigéria também revelou como a modernização foi uma época profundamente heterotópica para alguns. Para uma primeira geração de nacionalistas, foi um momento em que os profissionais se viam trabalhando na vanguarda de sua indústria e no auge de suas habilidades pessoais, e, assim, empurrando sua sociedade para o futuro. Seus esforços laborais produziram um senso de satisfação que tornou a memória da construção das infraestruturas de comunicação algo profundamente emocional. ${ }^{10}$

Ainda que grandes projetos infraestruturais possam ser usados para representar o poder do Estado aos seus cidadãos (Chalfin 2001, 2008, 2010; Harvey 2012; Limbert 2010; Mains 2012), os efeitos políticos desses projetos não podem ser simplesmente lidos a partir de sua superfície. Elas geram investimentos emocionais complexos que 
induzem a uma gama de respostas, às vezes contraintuitivas e provenientes de sensibilidades distintas, embora efêmeras. Khan (2006) descreve a construção da primeira autoestrada multipista paga no Paquistão, que levava à capital, Islamabad, e relata como o projeto criava um sentimento disjuntivo nos paquistaneses. Havia 'uma certa dessincronia entre a Autoestrada e o Paquistão', argumenta a autora, pois a autoestrada era tão racional e rápida que 'tornava o Paquistão muito ultrapassado'. Ela descreve um cenário complexo no qual a autoestrada representava, ao mesmo tempo, promessa e fracasso ${ }^{11}$. De Boeck (2011) descreve uma situação diferente, ainda que igualmente complexa, em que a desconstrução de um assentamento urbano em Kinshasa, capital da República Democrática do Congo, ocorre para abrir caminho para um condomínio de elite. Ele argumenta que o Estado 'travou uma guerra' contra os moradores desses assentamentos ao brutalmente destruir suas casas, enxergando nisso um clássico exemplo de violência do Estado contra aqueles cidadãos mais vulneráveis. No entanto, ele também relata que muitas das pessoas despejadas apoiavam o novo assentamento e viam nisso um senso de orgulho, mesmo que elas representassem exatamente o tipo de desordem que o desenvolvimento foi projetado para superar. "Sim, nós seremos as vítimas", comenta um pescador, "mas ainda assim será bonito" (De Boeck 2011:278). Vale a pena fazer uma pausa para considerar em que condições essa afirmação é possível, especialmente quando ela vai contra a narrativa aceita da modernização e suas vítimas, vivificando assim uma complexa mistura de desejo, fantasia e orgulho (ver também Humphrey 2005; Sneath et al 2009) para a qual a etnografia pode se abrir. E nos relembra como a profunda força da relação das pessoas com as infraestruturas - os sentidos de fascínio e pavor estimulados por elas - é uma importante parte de seu efeito político.

\section{A duplicidade da infraestrutura e a estética do direcionamento}

Houve, na antropologia africanista e nos estudos africanos, uma análise detalhada do processo de duplicidade, ou seja, de como siste- 
mas e práticas operam em divergência com seus supostos objetivos. Mbembe (2001) argumenta que o estilo de governo privado indireto que se desenvolve em muitos países africanos sob controle militar, mas que se estende muito além, opera por meio da constante proliferação de tecnologias formais do Estado - orçamentos, descrição de cargos, certificados - e da tomada de consciência de que estes têm pouca relação com realidade (ver Hull 2012 sobre documentos). Estados produzem outros tipos de objetos - estradas, fábricas, pontes - que também professam uma função técnica, mas que, na realidade, operam, ao mesmo tempo, em um nível diferente. Mbembe aponta que diversas vezes a função das concessões de projetos de infraestrutura tem muito mais relação com ganhar acesso aos contratos do governo e se beneficiar das redes de clientelismo do que com as funções técnicas. É por isso que estradas desaparecem, indústrias são construídas para nunca funcionarem e pontes não levam a lugar algum.

Jakobson identificou a poética como uma das seis diferentes funções presentes em qualquer ato verbal (emotiva, referencial, fática etc.). A função poética distingue-se das outras quando um ato verbal está organizado de acordo com as qualidades materiais do próprio significante, em vez do seu significado referencial. É quando a 'qualidade sensível dos signos' torna-se dominante, a função determinante de um ato verbal (Jakobson 1985) ${ }^{12}$. Assim, a poética é um rearranjo da hierarquia sobre a qual a significação é dominante em um evento discursivo. Discursos operam em muitos níveis simultaneamente, porém os atos verbais, em sua função poética, desprendem significados diferentes do que o fazem em suas funções referenciais ou emotivas.

No caso das infraestruturas, o modo poético demonstra como a forma se desprendeu das funções técnicas. É por meio das infraestruturas que o Estado oferece representações aos cidadãos e pede que eles as aceitem como fatos sociais, criando uma política do 'como se' (Wedeen 1999; ver também Apter 2005). O orçamento deve funcionar como se fosse uma representação realista, e ambos aqueles que a emitem e que a recebem agem como se fosse um documento significativo. 
Os orçamentos, no seu modo poético, tornam-se "atos simbólicos arbitrários” (Mbembe \& Roitman 1995:337), tanto quanto as técnicas de governabilidade. Essa noção é pertinente para qualquer lugar, mas assume uma intensidade particular em muitas partes do continente africano, onde a divulgação pública de uma ordem embasada no cálculo (por exemplo, tamanho de uma população, produto interno bruto etc.) é geralmente considerada como arbitrária. Como Mbembe apontou, ali as descrições oficiais dos cargos não correspondem aos poderes reais, os cargos são atribuídos por motivos não relacionados à qualificação profissional e a execução dos orçamentos tem pouco a ver com a sua elaboração.

Nesses momentos, o Estado é simultaneamente presente e ausente. Em seu texto/ensaio 'When a Pipe is not a Pipe' / 'Quando um cano não é um cano', Lea \& Pholeros (2010) descrevem exatamente esse processo na sua discussão sobre habitação entre os aborígenes, em que o que parece ser habitação não o é de fato. Há casas com canos que não se conectam com o esgoto e quartos somente parcialmente completos. Esses são "enganos compósitos”, como nomeiam os autores (Lea \& Pholeros 2010:191), que geram uma específica 'ordem estética'. Nesse sentido, não se trata de um cano em si, mas de sua produção como uma forma representacional, que permite que relatórios sejam escritos, orçamentos sejam cumpridos e patrocinadores tranquilizados. Um cano pode não estar ligado a um sistema de eliminação de efluentes, mas está ligado a técnicas de regulação, auditoria e administração. Sua forma material é transposta de um cano vazio para dígitos em um orçamento e palavras em uma página, e todas essas formas - como tubo vazio, como números e como uma série de letras - são canos. Todas são diferentes formas de materialização de um cano que permitem com que ele se movimente por diferentes regimes circulatórios.

Pesquisas recentes sobre o governo soviético examinaram precisamente as maneiras como as regras funcionavam tanto como um projeto estético quanto político que constituía o cidadão a partir dos 
modos de endereçamentos (Groys 2011). 'O comunismo, em última instância, criou estruturas estéticas efetivas e estruturas econômicas defeituosas', argumenta o estudioso de história da arte Vladimir Todorov (1994; ver também Humphery 2005). Ele se refere ao fato de que a efetividade técnica das fábricas soviéticas para produzir mercadorias era limitada, porém, seu poder político de treinar os sujeitos em uma relação particular com o poder estatal era extensivo, organizando as estruturas administrativas, preservando esse poder e o representando às pessoas por meio dos objetos da fábrica. Sob o comunismo, argumenta Todorov, "fábricas não são construídas para produzirem mercadorias, [mas] significados simbólicos [...] O resultado era um déficit de mercadorias e uma superprodução de significados simbólicos” (Todorov 1994:10).

Todorov aqui captura algo que precisa ser considerado. As infraestruturas operam em múltiplos níveis simultaneamente. Eles executam funções técnicas (movem tráfego, água ou eletricidade), mediando trocas à distância e conectando pessoas e coisas em sistemas heterogêneos e complexos. Além disso, operam como formas entextualizadas que têm relativa autonomia de suas funções técnicas. Para conceber essa operação enquanto uma forma poética nos termos de Jakobson, é preciso rearranjar a hierarquia das funções de modo que a dimensão estética (em vez da dimensão técnica) seja dominante.

Assim, muitos estudos que começam apontando como as infraestruturas são invisíveis até que falhem são imprecisos. Infraestruturas são objetos meta-pragmáticos, signos de si mesmos mobilizados em regimes circulatórios para estabelecerem um arranjo de efeitos. Em quase todos os estudos sobre infraestrutura é um lugar comum e aparentemente obrigatório repetir a afirmação da Star de que as infraestruturas são "invisíveis por definição", tomadas por certas até que "se tornam visíveis com a quebra” (Star 1999:380). Porém essa afirmativa é parcialmente verdadeira e completamente insustentável para tratar da infraestrutura como um todo. Invisibilidade é certamente um aspecto da infraestrutura, mas não é o único dentro da extrema varieda- 
de de visibilidades, do não visto aos grandes espetáculos e tudo o que está no meio.

A literatura antropológica descreve uma variedade de maneiras nas quais a infraestrutura é muitas vezes qualquer coisa menos invisível. Sneath (2009) argumenta que a eletricidade na Mongólia era um símbolo de Estado tão importante que era então chamada de 'a luz de Lenin'. Essa visão não é distante de Anand, para quem um ativista das comunidades pobres de Mumbai poderia facilmente narrar uma história do abastecimento de água revelando um conhecimento técnico impressionante; em realidade, os dois canos da comunidade (e suas dimensões) eram chamados pelos nomes dos políticos que os forneceram (i.e., cano duas polegadas de diâmetro do Partido do Congresso [Congress Party] e cano nove polegadas de diâmetro Shiv Sena). Para que esse sistema de clientelismo funcionasse ou para a modernidade infraestrutural da encenação de Lenin ter sucesso, a visibilidade era necessária para renovar continuamente o seu efeito político. Whinter (2008) nota como os habitantes de uma zona rural de Zanzibari tinham um conhecimento altamente sofisticado sobre o consumo de energia de cada utensílio que eles possuíam, e Von Schnitzler cita como os engenheiros municipais referiam-se aos residentes de Soweto enquanto especialistas em tecnologia por suas habilidades de desmontar, remontar e raquear os hidrômetros. $\mathrm{O}$ amplo reconhecimento da profundidade e a sofisticação do conhecimento técnico indicam a impossibilidade de essas tecnologias desvanecerem-se no cenário; de fato, como argumenta Carse (2012), toda visibilidade é situada, e o que é cenário para uma pessoa, é objeto de preocupação diária para outra. A questão não é afirmar isto ou aquilo como uma condição inerente das infraestruturas, mas examinar como a (in)visibilidade é mobilizada e o porquê. Afirmações genéricas sobre a invisibilidade das infraestruturas não se sustentam. Talvez o exemplo mais marcante dessa hipervisibilidade seja a descrição de Baker da cerimônia de Suharto envolvendo o lançamento do satélite Palapa. Suharto segurava uma kris, uma adaga cerimonial utilizada por monarcas javaneses 
como símbolo de seu regime. A adaga estava incrustada com 17 pedras preciosas (17 referindo-se à data de independência da Indonésia), e o botão para lançar o satélite estava incrustado como uma das pedras. Barker argumenta que Suharto usou o "satélite enquanto um tipo de kris moderna [...] para unificar o arquipélago sob seu regime" (Baker 2005:706). Seria difícil pensar em uma infraestrutura mais rica e vivida a nível simbólico do que essa.

\section{Materialidade infraestrutural e a produção da experiência do ambiente}

Uma segunda dimensão, relativamente oposta à estética, oferece outra profícua maneira de pensar as infraestruturas, partindo de um conceito mais antigo de Aristóteles, aisthesis (Buck-Morss 1992; Meyer 2009; Verrips 2006). Aisthesis não se refere à apreciação mental de uma obra de arte, mas à reação do corpo à realidade vivida: "É uma forma de cognição, alcançada por meio do paladar, do tato, da escuta, da visão e do olfato" argumenta Buck-Morss (1992:6). Nesse sentido, a estética não é uma representação, mas uma experiência materializada, que é governada pelas maneiras com que as infraestruturas produzem as condições do ambiente da vida cotidiana: nossos sensos de temperatura, velocidade, florescência, assim como as ideias que associamos com essas situações. As infraestruturas criam uma sensitividade da modernidade, um processo no qual tanto corpo quanto mente apreendem o que é ser moderno, mutável e progressivo.

Para Mrázek, discutindo sobre o domínio colonial alemão na Indonésia, a infraestrutura não é somente um objeto técnico, mas uma linguagem a ser aprendida, um meio de sintonizar-se com o desejo e o senso de possibilidade expressos na própria materialidade da infraestrutura. De certa maneira, alinha-se com o argumento de Barry (2001) de que uma sociedade tecnológica é aquela que cultiva as habilidades e os conhecimentos técnicos de seus cidadãos como uma condição de funcionamento em um mundo moderno ${ }^{13}$. Porém Mrázek analisa esse processo em termos profundamente estéticos, focando sobre como os 
materiais da infraestrutura - a solidez da estrada, a intensidade de sua escuridão e a suavidade do acabamento - produzem experiências políticas e sensoriais. "A limpeza das estradas, nessa lógica," argumenta, "era o momento da pureza, até mesmo da democracia" (Mrázek 2002:8). A construção da infraestrutura colonial foi a imposição de estradas sólidas - de ferro para trens, de asfalto para carros - sobre os caminhos sujos, lamacentos e suaves da Indonésia. São propriedades materiais surpreendentemente novas e diferentes, com seus próprios tipos de acidentes e maneiras de redirecionar as relações físicas e sociais. E eles são uma linguagem que os outros precisam aprender.

Os materiais da infraestrutura são chave na análise de Mrázek. A estrada, enquanto uma expressão do caráter alemão, não era somente limpa, mas era uma limpeza continuamente ameaçada pela poeira. Soprada quando as fezes dos cavalos e búfalos secavam no sol quente e marcada pelos pés sujos de lama dos pedestres nativos: essa sujeira ameaçava amolecer a dureza da estrada. Esse problema era parte de uma complicação ainda maior para os alemães: Havia demasiada suavidade nas colônias, onde até mesmo o ar parecia conter água. A chuva tropical encharcava, enfraquecia e erodia as estradas. "Aonde quer os nativos fossem, especialmente quando ousavam se aproximar de uma estrada moderna, eles eram lidos como portadores dessa suavidade consigo, nas suas línguas, em seus pés, em suas rodas" (Mrázek 2002:27).

As infraestruturas operam no nível da superfície, o que BuckMorss (1992) se refere como o terminae da parte externa do corpo pele, nariz, olho, orelha - em vez da mente, que é interna. Suavidade, dureza, o som da cidade, a sensação de calor ou frio, todas são experiências reguladas pelas infraestruturas e têm amarrações conceituais mais amplas, como argumenta Mrázek.

Mumford (2010) divide a história em períodos tecnológicos e argumenta que cada era privilegia uma tecnologia que a representa. $\mathrm{Na}$ era industrial, o ferro decisivamente substituiu a madeira, o primeiro material utilizado para construir - junto com todos os resíduos de um 
passado orgânico rural. "Uma pessoa foi dormir numa cama de ferro e lavou o rosto numa [...] cuba de ferro [...]; senta-se no fundo de uma locomotiva de ferro e segue pela estrada com trilhos de ferro, passa sobre uma ponte de ferro e chega em uma estação de trem coberta de ferro" (Munford 2010:164). O ferro tem propriedades específicas. Enquanto as pedras resistem à compressão e são aglomeradas em pilhas, o ferro pode ser derretido e esticado para sustentar pesos enormes e criar espaços abertos elevados (Giedion 1995). Finos postes de ferro permitem a criação de vastas áreas para a exibição de mercadorias e para a circulação de grandes multidões. Esses aspectos são centrais para o surgimento de novos espaços para o capital: estações de trem, lojas de departamento e salões de exposição (Giedion 1995; Benjamin 1999). Há uma afinidade entre o processo industrial que produz o ferro, a estética dos espaços que o material pode trazer à existência e as novas formas de exibição, primordiais para a sociedade do consumo. Qualquer pessoa caminhando por uma estação de trem ou subindo em um elevador de uma loja de departamento estava se movendo em um espaço formado e capturado pelo industrialismo (Benjamin 1999; Giedion 1995; Marx 1990).

Schivelbusch (1995) observa que a mudança da chama viva para a iluminação incandescente representa a substituição de uma fonte de luz oscilante, variável e dinâmica por outra rígida e estável. Ele argumenta que essa mudança produziu uma transformação sutil na percepção do cotidiano no mundo, ainda que totalizante. Otter (2008) relembra esse sentimento em seu relato sobre a construção da primeira estrada de asfalto em Londres em 1869, e a reação que ela produziu. Para os contemporâneos, isso criou um efeito quase misterioso de silenciosidade. Era como se elas estivessem isolando a cidade do barulho das rodas das carruagens sobre o calçamento. O concreto é outro material de construção universal com qualidade táteis específicas que moldam o ambiente de vida. Harvey (2010) argumenta esse ponto em relação ao caso da construção de estradas no Peru, o concreto esfregado coloca em xeque as concepções andinas de desidratação e vitalida- 
de. $\mathrm{O}$ concreto era algo considerado distinto por ser uma substância mutável, porém irreversivelmente dura ao secar (Taussig 2004). Essas ideias estavam operando na região andina, onde técnicas de desidratação (mumificação) foram centrais para armazenar a vitalidade do morto (Harvey 2010:38). Harvey argumenta que a dureza do concreto questionava certezas cosmológicas fundantes acerca de pedras, terra, rochas e montanhas.

Os materiais de infraestrutura - ferro, barro, cabos de fibra óptica, plástico - tanto representam uma era, no sentido de como o ferro era um material exemplar no século XIX, quanto provocam uma apreensão sensorial da existência. Essa é a sua dimensão estética. Ranciere (2006, 2009) vê a estética como uma propriedade da arte e concede a ela o potencial crítico de reconfigurar o território do comum e permitir a inserção de novas vozes no espaço político. Mas quase um século antes, Giedion (1995) dissociou esse atributo da arte e o atribuiu às infraestruturas que o sustentam. Para ele, a revolução nos materiais de construção, impulsionada pela ascensão do concreto e do ferro, significou que o ferro 'demoliu' a arquitetura. Os materiais infraestruturais e as forças de produção que eles materializam ultrapassaram a arquitetura, revelando sua base naturalizada, oferecendo uma revelação do estado das coisas e modelando a emergência dos sujeitos modernos. "As construções do século XIX desempenham um papel subconsciente importante. Externamente, a construção ainda ostenta a antiga força persuasiva; por baixo, oculto por trás de fachadas, a base de nossa atual existência está tomando forma" (Giedion 1995:87; ver também Benjamin 1999). Fennell (2011), examinando as especificidades dos vários sistemas de aquecimento das habitações públicas em Chicago, concebe essa infraestrutura como uma 'política sensorial'. 'O Projeto Aquecer', como chamavam os residentes, referia-se ao constante alto nível de calor que os antigos moradores desfrutavam antes de suas residências serem destruídas e eles serem realocados. Como disse um senhor idoso a Fennell, "Nós tínhamos verão no inverno" (Fennell 2011:50), de modo que o 'Projeto Aquecer' representava um tipo de 
essência platônica dos sistemas de calefação. Fennell argumenta que isso também se tornou o fundamento para avaliar os benefícios, os riscos e as consequências da destruição e da construção de habitações públicas em uma era liberal. Para Fennell, o aquecimento é uma experiência sensorial e afetiva por meio da qual a subjetividade política é encenada e as reivindicações por reconhecimento político expressas.

\section{Conclusões}

Pelo fato de os objetos básicos das infraestruturas serem tão diversos e poderem ser analisados de maneiras diferentes, a escolha metodológica é necessariamente uma questão teórica. As infraestruturas são sistemas tecnológicos e a maneira de compreendê-los seria um processo de análise de máquinas conectadas em rede? São elas os instrumentos financeiros, práticas de contabilidade e orçamentárias, estruturas administrativas ou técnicas organizacionais? São elas biológicas, incorporadas na fisicalidade dos homens que usam seus tamanhos, massa e atitude para atrair lances no pregão em Chicago (Zaloom 2003, 2006)? Ou elas são sociais, compostas de práticas de visitar, beber um chá e cumprimentar, ou seja, investimentos em socialidade que podem ser recompensados por criarem uma teia de relações necessárias para todos os tipos de atividades social, econômica e política (Elyachar 2010, 2011; Simone 1998, 2001, 2004)? Os estudos de infraestrutura tendem a privilegiar a dimensão tecnológica, mesmo que qualifiquem isso por meio de uma definição dos espaços urbanos como sistemas híbridos de humanos e máquinas amarrados pelas redes infraestruturais. Ainda assim, um dos aspectos mais dinâmicos das pesquisas antropológicas recentes sobre infraestrutura é a absoluta diversidade de modos de conceber e analisar as infraestruturas, que, cumulativamente, apontam para uma instabilidade da produção das unidades básicas da pesquisa.

Aqui mal pude discutir algumas abordagens das infraestruturas. Deleuze (1992) argumenta - não diferente de Mumford - que certos tipos de máquinas podem ser associados a certos tipos de sociedade; 
e se o século XIX foi construído sobre tecnologias industriais de contenção, os séculos XX e XXI estão construídos sobre estruturas de controle mediadas por computadores. Essa é uma virada cibernética decisiva que só recentemente foi tomada seriamente na antropologia pelo estudo de Fisch (2013) sobre o sistema de metrô de Tokyo. Nessa sociedade, a produção tem sido terceirizada, o valor produzido virtualmente e as infraestruturas localizam-se em protocolos de computador e formas de captura que estão projetadas em plataformas de mídias sociais, tais como Facebook e Google. Nessa economia, a regulação da atenção é central para a produção de valor (Chun 2008; Galloway 2006). Outra pesquisa examina a infraestrutura midiática de ativismo, sua arquitetura e práticas de circulação por meio das quais ideias políticas são codificadas em formas midiáticas, materializadas em campanhas e, então, levadas ao público (Keenan \& Weizman 2012; Latour \& Weibel 2005; McLagan 2006, 2008; McLagan \& McKee 2012). Pode-se apontar ainda outras literaturas sobre planejamento e espaço, numerosas demais para listar. Mas a mídia representa um caso particularmente convincente, haja vista sua centralidade para a produção de formas emergentes de valor.

Beller (2006) discute como a ascensão do cinema representou uma transformação na produção de valor, distanciando-a de uma economia industrial em direção ao estímulo de desejo e, com essa mudança, de consumo. "Em vez de exigir que um Estado construa as estradas que permitam a circulação de suas mercadorias, como o fez Ford, o cinema constrói seus meios de circulação diretamente nos olhos e nas sensorialidades de seus espectadores. São os telespectadores que performam o trabalho que abre caminhos para novas mercadorias" (Beller 2006:209). Se isso foi verdade para a sociedade moderna, é algo que tem se multiplicado na nossa era das mídias sociais, em que controle e valor estão indissoluvelmente ligados com os conjuntos de máquinas que constituem as infraestruturas digitais contemporâneas. Para tal tarefa, novos enquadramentos teóricos e etnográficos têm ainda de serem inventados na antropologia. 


\section{Notas:}

1 Nota do autor: Agradeço a Meg McLagan e Peter Redfield pelas leituras deste artigo. Estendo a minha gratidão a duas turmas de pós-graduação sobre a poética e política de infraestrutura em que muitas destas ideias foram debatidas. Agradeço a todos estes estudantes pelas suas contribuições. $\mathrm{O}$ autor não tem conhecimento de qualquer filiação, filiação, financiamento, ou participações financeiras que possam afetar a objetividade deste artigo. Nota dos tradutores: agradecemos a Brian Larkin por seu entusiasmo com a proposta de tradução e seu suporte no processo de concessão dos direitos autorais do presente artigo.

2 Este artigo é uma tradução da versão original em inglês, cujo título é 'The politics and poetics of infrastructure'. Foi publicado pela primeira vez em Annual Review of Anthropology, 42:327-343, 2013, https://doi.org/10.1146/annurev-anthro-092412-155522.

3 Nota dos tradutores: O termo inglês 'breakdown' foi traduzido aqui com falhas para indicar seja pequenas quebras do sistema ou grandes colapsos. Todavia, ao longo do artigo, o termo é às vezes traduzido como colapso, quando evidentemente se refere a grandes falhas.

4 Apesar de a antropologia ter começado a analisar infraestrutura enquanto um conceito apenas recentemente (Anand 2011, 2012; Appel 2012a e b; Carse 2012; Chalfin 2010; Collier 2011; Collier \& Lakoff 2008; Dalakoglou \& Harvey 2012; Elyachar 2010, 2011; Harvey 2012; Harvey \& Knox 2012; Humphrey 2005; Mains 2012; Pedersen 2011; Sneath 2009; Sneath et al 2009; Von Schnitzler 2008; Winther 2008), a literatura há muito tem analisado tecnologias específicas. Nisso incluem-se barragens (Campregher 2010; Ghosh 2008; Mains 2012), carros (Chalfin 2008; Sanders 2008; Verrips \& Meyer 2001) e a excelente literatura sobre estrada (Dalakoglou 2010, 2012; Dalakoglou \& Harvey 2012; Harvey 2010; Harvey \& Knox 2012; Kernaghan 2012; Khan 2006; Knox \& Harvey 2011; Mains 2012; Manning 2012; Masquelier 1992, 2002, 2008; Morris 2010; Roseman 1996). A força desse trabalho pode ser percebida nos vários estudos recentes sobre projetos de infraestrutura que foram publicados em livros (Bear 2007; Limbert 2010; Winther 2008).

5 A análise de Fisch (2013) do metrô de Tokyo enquanto um conjunto tecnológico pós-cibernético é um importante trabalho sobre essa discussão.

6 Nota dos tradutores: No texto original são reportados os nomes das patentes do Edison: Jumbo Generator e Main and Feeder.

7 Nota dos tradutores: O termo inglês 'township' indica subúrbios de classe baixa, que foram criados na África do Sul, na época do apartheid, para segregar, com base racial, os trabalhadores.

8 Nota dos tradutores: este trecho de 'O Mal-Estar na Civilização' foi retirado da edição em português da Companhia das Letras de 2010, traduzida por Paulo César Lima de Souza.

9 Tal abordagem está bem representada na literatura antropológica (Barker 2005; Humphrey 2005; Khan 2006; Lea \& Pholerus 2010; Sneath 2009; Sneath et al 2009). 
10 Nota dos tradutores: geralmente o termo em inglês aqui utilizado é 'embodiement'. Esse termo, considerada a ampla influência das teorias de inspiração fenomenológica popularizadas pelo trabalho de Thomas Csordas, é geralmente traduzido no campo antropológico como 'incorporação', remetendo a uma quebra da dicotomização entre corpo e mente. Neste artigo utilizamos o termo 'materialização' para sublinhar a centralidade da dimensão material das infraestruturas, considerando que é um ponto central desta teoria

11 Um fantástico exemplo disso pode ser visto no projeto Leabanese Rocket Society dos artistas Joana Hadjithomas e Khalil Joreige. A sociedade foi formada na década de 1960, concomitante ao lançamento da Apollo e à primeira missão espacial Soviética tripulada, e representou uma tentativa por parte do Líbano/esforço libanês em desenvolver seu próprio programa de foguetes (ver Hadjithomas \& Joreige 2013). Hadjithomas \& Joreige veem tal projeto como um esforço fantástico de uma pequena nação em desenvolvimento, mas eles também levam a sério o empenho científico envolvido (para um projeto relacionado, ver Redfield 2000). Redfield argumenta que o desejo de imitar projetos tecnológicos como um meio de reivindicar a participação de uma nação na vanguarda da modernidade é um aspecto presente em todos os projetos espaciais. A única diferença é o grau com que um grupo se sente 'fazendo progresso' ou 'alcançando' os outros (comunicação pessoal de P. Redfield).

12 Limbert (2010) discute também os sentimentos de inquietação e disjunção que as infraestruturas modernas produzem. Ela argumenta que as infraestruturas produzidas com o dinheiro do petróleo geram uma sensação disjuntiva do tempo - o tempo do petróleo - em que a modernização deve ocorrer antes que o petróleo (e o seu tempo) acabe.

13 A rima na poesia é o exemplo paradigmático de Jakobson (1985). Quando uma palavra é selecionada num poema para rimar, Jakobson argumenta que sua função referencial (o que isto significa) é menos relevante do que sua relação homofônica com outra palavra (como isto soa). Assim, a poética chama atenção para a materialidade do significante. A rima na poesia é o exemplo paradigmático de Jakobson (1985). Quando uma palavra é selecionada num poema para rimar, Jakobson argumenta que sua função referencial (o que isto significa) é menos relevante do que sua relação homofônica com outra palavra (como isto soa). Assim, a poética chama atenção para a materialidade do significante.

14 A ideia de que uma infraestrutura é uma linguagem ou, mais comumente, um conjunto de competências culturais a serem aprendidas é explorada em diversos trabalhos (Harvey 2010; Khan 2006; Lea \& Pholerus 2010; von Schnitzler 2008; Winther 2008). Normalmente essas noções centram-se em torno do fracasso das pessoas de 'aprender linguagem' do modo de vida moderno (Lea \& Pholeros 2010). Essas acusações, que frequentemente são feitas por funcionários do governo e engenheiros, sugerem que as pessoas não sabem como cuidar das tecnologias que possuem. Isso sustenta o apontamento de Barry de que o tecnológico, longe da tecnologia por si, envolve modos de competência e disposição. 


\section{Referências:}

ANAND, N. 2011. "PRESSURE: The PoliTechnics of water supply in Mumbai". Cult. Anthropol., 26(4):542-64.

2012. "Municipal disconnect: on abject water and its urban infrastructures". Ethnography, 13(4):487-509.

APPEL, H. 2012a. "Offshore work: oil, modularity, and the how of capitalism in Equatorial Guinea”. Am. Ethnol., 39(4):692-709. . 2012b. "Walls and white elephants: oil extraction, responsibility, and infrastructural violence in Equatorial Guinea". Ethnography, 13:439-65.

APTER, A. 2005. The Pan-African Nation: Oil and the Spectacle of Culture in Nigeria. Chicago: Univ. Chicago Press.

ARCHAMBAULT, J. S. 2012. “'Travelling while sitting down': mobile phones, mobility and the communication landscape in Inhambane, Mozambique". Africa, 82(3):393-412.

BARKER, J. 2005. "Engineers and political dreams: Indonesia in the satellite age". Curr. Anthropol., 46(5):703-27.

BARRY, A. 2001. Political Machines: Governing a Technological Society. London: Continuum.

BEAR, L. 2007. Lines of the Nation: Indian Railway Workers, Bureaucracy, and the Intimate Historical Self. New York: Columbia Univ. Press.

BELlER, J. 2006. The Cinematic Mode of Production: Attention Economy and the Society of the Spectacle. Lebanon, NH: Dartmouth Univ. Press.

BENJAMIN, W. 1999. The Arcades Project. Cambridge, MA: Harvard Univ. Press.

BENNETT, J. 2010. Vibrant Matter: A Political Ecology of Things. Durham, NC: Duke Univ. Press.

BIJKER, W. E. 1997. Of Bicycles, Bakelites, and Bulbs: Toward a Theory of Sociotechnical Change. Cambridge, MA: MIT Press.

BIJKER, W. E., HUGHES, T.P. \& PINCH, T. J. 1987. The Social Construction of Technological Systems: New Directions in the Sociology and History of Technology. Cambridge, MA: MIT Press.

BOWKER, G. C. \& STAR S. L. 2000. Sorting Things Out: Classification and Its Consequences. Cambridge, MA: MIT Press.

BUCK-MORSS, S. 1992. "Aesthetics and anaesthetics: Walter Benjamin's artwork essay reconsidered". October, 62:3-41.

CALLON, M. 1986. "Some elements of a sociology of translation: domestication of the scallops and the fishermen". In LAW, J. (ed.): Power, Action and Belief: A New Sociology of Knowledge, pp. 67-83. London: Routledge \& Kegan Paul. (ed). 1998. Laws of the Markets. Oxford: Wiley-Blackwell.

CAMPREGHER, C. 2010. "Shifting perspectives on development: an actornetwork study of a dam in Costa Rica”. Anthropol. Q., 83(4):783-804. 
CARSE, A. 2012. "Nature as infrastructure: making and managing the Panama Canal watershed". Soc. Stud. Sci., 42(4):539-63.

CHALFIN, B. 2001. "Border zone trade and the economic boundaries of the state in north-east Ghana". Africa, 71(2):202-24.

2008. "Cars, the customs service, and sumptuary rule in neoliberal

Ghana". Comp. Stud. Soc. Hist., 50(2):424-53.

2010. Neoliberal Frontiers: An Ethnography of Sovereignty in West Africa.

Chicago: Univ. Chicago Press.

CHUN, W. H. K. 2008. Control and Freedom: Power and Paranoia in the Age of Fiber Optics. Cambridge, MA: MIT Press.

COLLIER, S. \& LAKOFF, A. 2008. "The vulnerability of vital systems: how 'critical infrastructure' became a security problem". In CAVELTY, M. D. \& KRISTENSEN, K. S. (eds.): The Politics of Securing the Homeland: Critical Infrastructure, Risk and Securitisation, pp. 40-62. New York: Routledge.

COLLIER, S. J. 2011. Post-Soviet Social: Neoliberalism, Social Modernity, Biopolitics. Princeton, NJ: Princeton Univ. Press.

DALAKOGLOU, D. 2010. "The road: an ethnography of the Albanian-Greek cross-border motorway". Am. Ethnol., 37(1):132-49.

.2012. “The road from capitalism to capitalism': infrastructures of (post) socialism in Albania". Mobilities, 7(4):571-86.

DALAKOGLOU, D. \& Harvey, P. 2012. "Roads and anthropology: ethnographic perspectives on space, time and (im)mobility". Mobilities, 7(4):459-65.

DE BOECK, F. 2011. "Inhabiting ocular ground: Kinshasa's future in the light of Congo's spectral urban politics”. Cult. Anthropol., 26(2):263-86.

DELEUZE, G. 1992. "Postscript on the societies of control". October, 59:3-7.

EDWARDS, P. N. 1997. The Closed World: Computers and the Politics of Discourse in Cold War America. Cambridge, MA: MIT Press. . 1998. "Y2K: Millennial reflections on computers as infrastructure". Hist. Tech., 15(1-2):7-29.

2003. "Infrastructure and modernity: force, time, and social organization in the history of so-ciotechnical systems". In MISA, T. J. \& BREY, P. (eds.): Modernity and Technology, pp. 185-225. Cambridge, MA: MIT Press.

EDWARDS, P. N. et al. 2009. "Introduction: an agenda for infrastructure studies". J. Assoc. Inf. Syst., 10(5):364-74.

ELYACHAR, J. 2010. "Phatic labor, infrastructure, and the question of empowerment in Cairo". Am. Ethnol., 37(3):452-64.

. 2011. "The political economy of movement and gesture in Cairo". J. R. Anthropol. Inst., 17(1):82-99.

FENNELL, C. 2011. "'Project heat' and sensory politics in redeveloping Chicago public housing". Ethnography, 12(1):40-64.

FERGUSON, J. 1999. Expectations of Modernity: Myths and Meanings of Urban Life on the Zambian Copperbelt. Berkeley: Univ. Calif. Press. 
FISCH, M. 2013. "Tokyo's commuter train suicides and the society of emergence. Cult. Anthropol., 28(12):320-43.

FOUCAULT, M. 2010. The Birth of Biopolitics: Lectures at the Collège de France, 1978-1979. New York: Picador.

2011. The Government of Self and Others: Lectures at the Collège de France, 1982-1983. New York: Picador.

FREUD, S. 1989. Civilization and Its Discontents. New York: Norton. 2010. "O mal-estar na civilização". In Freud, S.: Obras completas - volume 18, pp. 9-89. São Paulo: Companhia das Letras.

GALLOWAY, A. 2006. Protocol: How Control Exists After Decentralization. Cambridge, MA: MIT Press.

GANDY, M. 2008. "Landscapes of disaster: water, modernity and urban fragmentation in Mumbai”. Environ. Plann., 40:108-30.

GELBER, E. 2015. "Black Oil Business: Rogue Pipelines, Hydrocarbon Dealers, and the 'Economics' of Oil Theft". In APPEL, H. et al (ed.): Subterranean Estates: Life Worlds of Oil and Gas, pp. 274-290. Ithaca/ London: Cornell University Press.

GHOSH, K. 2008. "Between global flows and local dams: indigenousness, locality, and the transnational sphere in Jharkhand, India". Cult. Anthropol., 21(4):501-34.

GIEDION, S. 1969. Mechanization Takes Command: A Contribution to Anonymous History. New York: Norton

1995. Building in France, building in iron, building in ferroconcrete. Los Angeles: Getty Cent. Hist. Art Humanit.

GRAHAM, S., DESAI, R. \& MCFARLANE, C. 2013. "Water wars in Mumbai". Public Culture, 25(1):115-41.

GRAHAM, S. \& MARVIN, S. 1996. Telecommunications and the City: Electronic Spaces, Urban Places. London: Routledge.

2001. Splintering Urbanism: Networked Infrastructures, Technological Mobilities and the Urban Condition. London: Routledge.

GROYS, B. 2011. The Total Art of Stalinism: Avant-Garde, Aesthetic Dictatorship, and Beyond. New York: Verso

HADJITHOMAS, J. \& JOREIGE, K. 2013. On the Lebanese Rocket Society. (www.e-flux.com/ journal/on-the-lebanese-rocket-society-2/; acesso em $12 / 11 / 2020)$.

HANSEN, T. B. \& VERKAAIK, O. 2009. "Introduction-Urban charisma on everyday mythologies in the city". Crit. Anthropol., 29(1):5-26.

HARVEY, P. 2010. "Cementing relations: the materiality of roads and public spaces in provincial Peru”. Soc. Anal., 54(2):28-46.

. 2012. "The topological quality of infrastructural relation: an ethnographic approach". Theory Cult. Soc., 29(4-5):76-92. 
HARVEY, P. \& KNOX, H. 2012. "The enchantments of infrastructure". Mobilities, 7(4):521-36.

HECHT, G. (ed). 2011. Entangled Geographies: Empire and Technopolitics. Cambridge, MA: MIT Press.

HEIDEGGER, M. 1977. The Question Concerning Technology, and Other Essays. New York: Harper Torchbooks.

HUGHES, T. P. 1987. "The evolution of large technological systems". In BIJKER, W. E., HUGHES, T.P. \& PINCH, T. J. (eds.): The Social Construction of Technological Systems: New Directions in the Sociology and History of Technology, pp. 51-82. Cambridge, MA: MIT Press.

. 1993. Networks of Power: Electrification in Western Society, 1880-1930. Baltimore, MD: Johns Hopkins Univ. Press.

HULL, M. S. 2012. "Documents and bureaucracy". Annu. Rev. Anthropol., 41:251-67.

HUMPHREY, C. 2005. "Ideology in infrastructure: architecture and Soviet imagination". J. R. Anthropol. Inst., 11(1):39-58.

JACKSON, S. J. et al. 2007. Understanding infrastructure: history, heuristics, and cyberinfrastructure policy. (https://firstmonday.org/ojs/index.php/fm/ article/view/1904; acesso em 12/11/2020).

JAKOBSON, R. 1985. Closing statements: linguistics and poetics. In INNIS. R. E. (ed.): Semiotics: An Introductory Anthology, pp. 145-175. Bloomington: Indiana Univ. Press.

JOYCE, P. 2003. The Rule of Freedom: Liberalism and the Modern City. London: Verso.

KEENAN, T. \& WEIZMAN, E. 2012. Mengele's Skull: The Advent of Forensic Aesthetics. New York: Sternberg.

KERNAGHAN, R. 2012. "Furrows and walls, or the legal topography of a frontier road in Peru". Mobilities, 7(4):501-20.

KHAN, N. 2006. "Flaws in the flow: roads and their modernity in Pakistan". Soc. Text, 24(89):87-113.

KNOX, H. \& HARVEY, P. 2011. "Anticipating harm: regulation and irregularity on a road construction project in the Peruvian Andes". Theory Cult. Soc., 28(6):142-63.

KOCKELMAN, P. 2010. "Enemies, parasites, and noise: how to take up residence in a system without becoming a term in it". J. Ling. Anthropol., 20(2):406-21.

LARKIN, B. 2008. Signal and Noise: Media, Infrastructure, and Urban Culture in Nigeria. Durham, NC: Duke Univ. Press.

LATOUR, B. 1993. We Have Never Been Modern. Transl. C Porter. Cambridge, MA: Harvard Univ. Press. Press. 1996. Aramis, or the Love of Technology. Cambridge, MA: Harvard Univ. 2007. Reassembling the Social: An Introduction to Actor-Network-Theory. Oxford, UK: Oxford Univ. Press. 
LATOUR, B. \& WEIBEL, P. (eds). 2005. Making Things Public: Atmospheres of Democracy. Cambridge, MA: MIT Press.

LEA, T. \& PHOLEROS, P. 2010. "This is not a pipe: the treacheries of indigenous housing". Public Culture, 22(1):187-209.

LEFEBVRE, H. 1991. The Production of Space. Oxford, UK: Blackwell.

LIMBERT, M. 2010. In the Time of Oil: Piety, Memory, and Social Life in an Omani Town. Stanford, CA: Stanford Univ. Press.

LOMBARDI, G. 1999. Computer networks, social networks and the future of Brazil. PhD Diss. N.Y. Univ.

MAINS, D. 2012. "Blackouts and progress: privatization, infrastructure, and a developmentalist state in Jimma, Ethiopia”. Cult. Anthropol., 27(1):3-27.

MANNING, P. 2012. Strangers in a Strange Land: Occidentalist Publics and Orientalist Geographies in Nineteenth Century Georgian Imaginaries. Boston, MA: Acad. Stud. Press.

MARX, K. 1990. Capital: Volume 1: A Critique of Political Economy. New York: Penguin Classics.

MASQUELIER, A. 1992. "Encounter with a road siren: machines, bodies and commodities in the imagination of a Mawri healer". Vis. Anthropol. Rev., 8(1):56-69.

2002. "Road mythographies: space, mobility, and the historical imagination in postcolonial Niger". Am. Ethnol., 29(4):829-56.

2008. "Of headhunters and cannibals: migrancy, labor, and consumption in the Mawri imagination". Cult. Anthropol., 15(1):84-126.

MATTELART, A. 1996. The Invention of Communication. Minneapolis: Univ. Minn. Press. 2000. Networking the World, 1794-2000. Minneapolis: Univ. Minn. Press.

MBEMBE, A. 2001. On the Postcolony. Berkeley: Univ. Calif. Press.

MBEMBE, A. \& ROITMAN, J. 1995. "Figures of the subject in times of crisis". Public Culture, 7(2):323-52.

MCLAGAN, M. 2006. "Introduction: Making human rights claims public". Am. Anthropol., 108(1):191-95.

. 2008. "Principles, publicity, and politics: notes on human rights media". Am. Anthropol., 105(3):605-12.

MCLAGAN, M. \& MCKEE, Y. (eds). 2012. Sensible Politics: The Visual Culture of Nongovernmental Activism. New York: Zone Books.

MEYER, B. 2009. Aesthetic Formations: Media, Religion, and the Senses. New York: Palgrave.

MITCHELL, T. 2002. Rule of Experts: Egypt, Techno-Politics, Modernity. Berkeley: Univ. Calif. Press. 2011. Carbon Democracy: Political Power in the Age of Oil. New York: Verso. 
MORRIS, R. C. 2010. "Accidental histories, post-historical practice?: re-reading Body of Power, Spirit of Resistance in the actuarial age”. Anthropol. Q., 83(3):581624.

MRÁZEK, R. 2002. Engineers of Happy Land: Technology and Nationalism in a Colony. Princeton, NJ: Princeton Univ. Press.

MUMFORD, L. 2010. Technics and Civilization. Chicago: Univ. Chicago Press.

OTTER, C. 2008. The Victorian Eye: A Political History of Light and Vision in Britain, 1800-1910. Chicago: Uni. Chicago Press.

PEDERSEN, M. A. 2011. Not Quite Shamans: Spirit Worlds and Political Lives in Northern Mongolia. Ithaca, NY: Cornell Univ. Press.

RANCIERE, J. 2006. The Politics of Aesthetics: The Distribution of the Sensible. London: Continuum. . 2009. Aesthetics and Its Discontents. London: Polity.

REDFIELD, P. 2000. Space in the Tropics: From Convicts to Rockets in French Guiana. Berkeley: Univ. Calif. Press.

ROSEMAN, S. R. 1996. "'How we built the road': the politics of memory in rural Galicia”. Am. Ethnol., 23(4):836-60.

SANDERS, T. 2008. "Buses in Bongoland: seductive analytics and the occult". Anthropol. Theory, 8(2):107-32

SCHIVELBUSCH, W. 1995. Disenchanted Night: The Industrialization of Light in the Nineteenth Century. Berkeley: Univ. Calif. Press.

SIMMEL, G. 1972. Georg Simmel on Individuality and Social Forms. Chicago: Univ. Chicago Press.

SIMONDON, G. 2016. On the Mode of Existence of Technological Objects. Minneapolis, MN: Univocal Pub.

SIMONE, A. 1998. "Globalization and the identity of African urban practices." In JUDIC, H. \& VLADISAVIC [, I. (eds.): Blank-Architecture, Apartheid and After, pp. 173-187. Rotterdam: NAi. . 2001. "On the worlding of African cities". Afr. Stud. Rev., 44:15-41. 2004. "People as infrastructure: intersecting fragments in Johannesburg". Public Culture, 16(3):407-29

SNEATH, D. 2009. "Reading the signs by Lenin's light: development, divination and metonymic fields in Mongolia". Ethnos, 74(1):72-90.

SNEATH, D., HOLBRAAD, M. \& PEDERSEN, M. A. 2009. "Technologies of the imagination: an introduction". Ethnos, 74(1):5-30

STAR, S. L. 1999. "The ethnography of infrastructure". Am. Behav. Sci., 43(3):37791.

STAR, S. L. \& RUHLEDER, K. 1996. "Steps toward an ecology of infrastructure: design and access for large information spaces". Inf. Syst. Res., 7(1):111-34.

STIEGLER, B. 1998 [1994]. Technics and Time, 1: The Fault of Epimetheus. Stanford, CA: Stanford Univ. Press. 
TAUSSIG, M. 2004. My Cocaine Museum. Chicago: Univ. Chicago Press.

TODOROV, V. 1994. Red Square, Black Square: Organon for Revolutionary Imagination. Albany: State Univ. New York Press.

VERRIPS, J. 2006. "Aisthesis and an-aesthesia". In LOVGREN, O. \& WILK, R. (eds.): Off the Edge: Experiments in Cultural Analysis, pp. 29-36. Copenhagen: Mus. Tusculanem.

VERRIPS, J. \& MEYER, B. 2001. "Kwaku's car: the struggles and stories of a Ghanaian long-distance taxi-driver”. In MILLER, D. (ed.): Car Cultures, pp. 153-84. Oxford: Berg

VON SCHNITZLER, A. 2008. "Citizenship prepaid: water, calculability, and techno-politics in South Africa”. J. South. Afr. Stud., 34(4):899-917

WEDEEN, L. 1999. Ambiguities of Domination: Politics, Rhetoric, and Symbols in Contemporary Syria. Chicago: Univ. Chicago Press.

WINTHER, T. 2008. The Impact of Electricity: Development, Desires and Dilemmas. New York: Berghahn.

YATES, J. 1993. Control Through Communication: The Rise of System in American Management. Baltimore: Johns Hopkins Univ. Press.

ZALOOM, C. 2003. "Ambiguous numbers: trading technologies and interpretation in financial markets". Am. Ethnol, 30(2):258-72. 2006. Out of the Pits: Traders and Technology from Chicago to London. Chicago: Univ. Chicago Press.

\begin{abstract}
Infrastructures are material forms that allow for the possibility of exchange over space. They are the physical networks through which goods, ideas, waste, power, people, and finance are trafficked. In this article I trace the range of anthropological literature that seeks to theorize infrastructure by drawing on biopolitics, science and technology studies, and theories of technopolitics. I also examine other dimensions of infrastructures that release different meanings and structure politics in various ways: through the aesthetic and the sensorial, desire and promise.
\end{abstract}

Keywords: Infrastructure, Technopolitics, Biopolitics, Aesthetics, Technology.

Tradução: Yuri Rosa Neves \& Alex Vailati.

Recebido em outubro do 2020. Aprovado em dezembro de 2020. 